\title{
Commentary: All tissue valves fail
}

\author{
David G. Lehenbauer, MD, and John Calhoon, MD
}

From the Division of Congenital Heart Surgery, Department of Cardiothoracic Surgery, UT Health San Antonio, San Antonio, Tex.

Disclosures: Authors have nothing to disclose with regard to commercial support.

Received for publication June 19, 2019; accepted for publication June 19, 2019; available ahead of print July 29, 2019.

Address for reprints: David G. Lehenbauer, MD, Department of Cardiothoracic Surgery, UT Health San Antonio, 7703 Floyd Curl Dr, San Antonio, TX 78229-3900 (E-mail: Lehenbauer@uthscsa.edu).

J Thorac Cardiovasc Surg 2020;159:1072-3

0022-5223/\$36.00

Copyright (C) 2019 by The American Association for Thoracic Surgery

https://doi.org/10.1016/j.jtcvs.2019.06.048

Modern-day bioprosthetic valves have many positive attributes. Just as all bleeding stops, however, all tissue valves fail. As a result, surgeons continue the quest for the perfect valve. Ideally, a valve should be viable, durable, nonthrombogenic, biologically inert, easily implanted with minimal invasiveness, resistant to infection, and able to grow with the patient-and, while we are at it, cheap and available on the shelf. In short, we search for a unicorn.

In this issue of the Journal, Pragt and colleagues ${ }^{1}$ present their experience with the implantation of the CarpentierEdwards (CE) Perimount valve (Edwards Lifesciences, Irvine, Calif). This is a retrospective review of 45 patients, almost exclusively adults, who underwent pulmonary valve replacement with a mean follow-up approaching 6 years. Previous early to intermediate experience with the CE Perimount valve in the pulmonary position has varied. Nomoto and associates ${ }^{2}$ reported a laudable reintervention rate of $0 \%$ for the first 5 years after valve implantation. Kwak and colleagues, ${ }^{3}$ however, demonstrated that $50 \%$ of the $\mathrm{CE}$ Perimount valves needed reintervention at 5 years. However, especially when implanted in children. ${ }^{3}$ In Pragt and colleagues' series, ${ }^{1}$ the number of patients with longer term follow-up was limited, but at 10 years, $83 \%$ of patients remained free from reintervention. Somewhat concerning is the finding that $11 \%$ of their patients had an episode of endocarditis. It is quite intriguing that only 1 of the 5 patients with endocarditis required reoperation. This nonoperative management suggests that prosthetic pulmonary valve endocarditis is far more responsive to medical therapy than seen in the traditional experience with endocarditis of the systemic prosthetic valve.

Pragt and colleagues' review ${ }^{1}$ of their experience with pulmonary valve replacement is unique in that they focused on a single product line of valves, performed morphologic examination of explanted valves, and chose a broader definition of failure to analyze their outcomes. They arbitrarily defined failure as "at least moderate regurgitation or moderate stenosis." ${ }^{1}$ This definition led to 12 patients, in a relatively short period, being designated as having valve failure

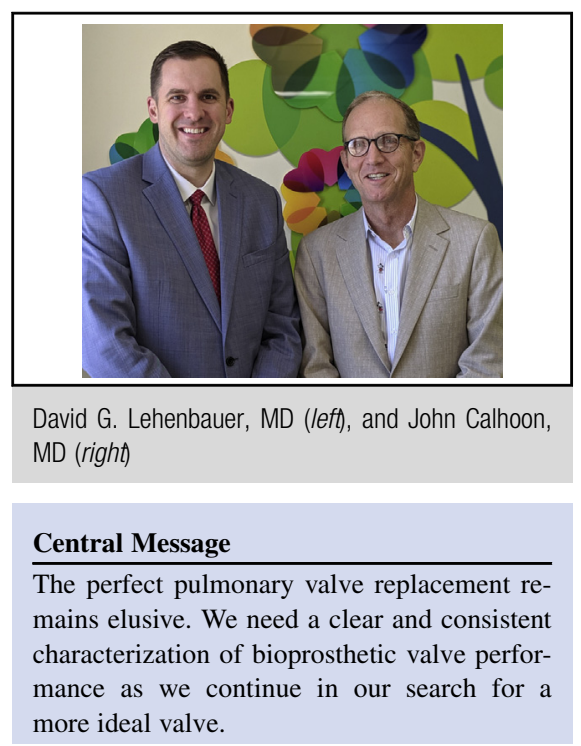

See Article page 1063.

for moderate stenosis or regurgitation. Previous studies have reserved the label of valve failure for those requiring reintervention on the valve and reported regurgitation or stenosis not requiring intervention as dysfunction. If some patients in this "failure" cohort were free of symptoms, should their moderate stenosis or regurgitation represent valve failure? This article highlights a need for consistent definition of bioprosthetic valve failure as we analyze patient outcomes in a search for a more nearly ideal valve.

A positive characteristic of stented bioprosthetic valves is that they facilitate percutaneous intervention with a valvein-valve approach. It should be noted that only 2 of 7 valves replaced in this cohort underwent a transcatheter approach. We know 1 of these 7 replacements was due to endocarditis; for the remainder, however, it is unclear why more of these patients did not undergo percutaneous replacement. Across all centers, we anticipate that the number of patients who undergo percutaneous valve replacement in the setting of a failed bioprosthetic valve will only continue to grow. Pragt and colleagues ${ }^{1}$ are to be commended for a nice series with reasonable outcomes in adult stented bovine pulmonary valve replacement. Their results, however, demonstrate that the ideal replacement for the pulmonary valve remains elusive.

\section{References}

1. Pragt H, Schoots MH, Accord RE, Arrigoni SC, Berger RM, Mariani MA, et al. A stented bovine pericardial prosthesis in the pulmonary position. J Thorac Cardiovasc Surg. 2020;159:1063-71.e1. 
2. Nomoto R, Sleeper LA, Borisuk MJ, Bergerson L, Pigula FA, Emani S, et al. Outcome and performance of bioprosthetic pulmonary valve replacement in patients with congenital heart disease. J Thorac Cardiovasc Surg. 2016;152:1333-42.e3.
3. Kwak JG, Lee JR, Kim WH, Kim YJ. Mid-term results of the Hancock II valve and Carpentier-Edward Perimount valve in the pulmonary portion in congenital heart disease. Heart Lung Circ. 2010;19:243-6. 